\title{
NTRK1 fusions for the therapeutic intervention of Korean patients with colon cancer
}

\author{
Do Youn Park ${ }^{1, *}$, Chan Choi ${ }^{3, *}$, Eunji Shin ${ }^{8}$, Jae Hyuk Lee ${ }^{3}$, Chae Hwa Kwon ${ }^{1}$, Hong- \\ Jae Jo ${ }^{2}$, Hyeong-Rok Kim${ }^{4}$, Hyun Sung Kim², Nahmgun Oh${ }^{2}$, Ji Shin Lee ${ }^{3}$, Ok Ku \\ Park $^{8}$, Eok Park ${ }^{8}$, Jonghoon Park, Jong-Yeon Shin ${ }^{5}$, Jong-Il Kim ${ }^{5,6}$, Jeong-Sun \\ Seo ${ }^{5,6,7}$, Hee Dong Park ${ }^{8}$ and Joonghoon Park ${ }^{8}$ \\ ${ }^{1}$ Department of Pathology, Pusan National University Hospital and Pusan National University School of Medicine, and \\ BioMedical Research Institute, Pusan National University Hospital, Busan, Republic of Korea \\ ${ }^{2}$ Department of Surgery, Pusan National University Hospital and Pusan National University School of Medicine, and BioMedical \\ Research Institute, Pusan National University Hospital, Busan, Republic of Korea \\ ${ }^{3}$ Department of Pathology, Chonnam National University Hwasun Hospital, Hwasun-gun, Jeollanam-do, Republic of Korea \\ ${ }^{4}$ Department of Surgery, Chonnam National University Hwasun Hospital, Hwasun-gun, Jeollanam-do, Republic of Korea \\ ${ }^{5}$ Genomic Medicine Institute (GMI), Medical Research Center, Seoul National University, Seoul, Republic of Korea \\ ${ }^{6}$ Department of Biochemical and Molecular Biology, Seoul National University College of Medicine, Seoul, Republic of Korea \\ ${ }^{7}$ Macrogen Inc., Seoul, Republic of Korea \\ ${ }^{8}$ LG Life Sciences Ltd., R\&D Park, Daejeon, Republic of Korea \\ * These authors have contributed equally to this work
}

Correspondence to: Joonghoon Park, email: joonghoon@lgls.com

Keywords: NTRK 1 fusion, Korean colon cancer, RNA-seq, predictive biomarker, targeted therapy

Received: September 11,2015 Accepted: December 07,2015 Published: December 22, 2015

\section{ABSTRACT}

The identification and clinical validation of cancer driver genes are essential to accelerate the translational transition of cancer genomics, as well as to find clinically confident targets for the therapeutic intervention of cancers. Here we identified recurrent LMNA-NTRK1 and TPM3-NTRK1 fusions in Korean patients with colon cancer ( 3 out of $147,2 \%$ ) through next-generation RNA sequencing (RNA-seq). NTRK1 fusions were mutually exclusive oncogenic drivers of colon cancer that were accompanied with in vitro potential of colony formation and in vivo tumorigenicity comparable to KM12, a human colon cancer cell line harboring TPM3-NTRK1 fusion. NTRK1-encoded TrkA protein was prevalent in 11 out of 216 Korean (5.1\%) and 28 out of 472 Chinese patients (5.9\%) from independent cohorts, respectively. The expression level of TrkA was significantly correlated with NTRK1 fusion $(p=0.0192)$, which was verified by a fluorescence in situ hybridization (FISH). Korean patients with TrkA-positive colon cancer had a marginal but significant shorter overall survival time than TrkA-negative colon cancer [hazard ratio $(H R)=0.5346,95 \%$ confidential interval $(\mathrm{CI})=0.2548-0.9722, p=0.0411]$. In addition, $\mathrm{KM12}$ cell line was sensitive to selective TrkA inhibitors. These results demonstrate that NTRK1 fusion is granted as a clinically relevant target for therapeutic intervention of colon cancer.

\section{INTRODUCTION}

Colorectal cancer (CRC) is the third most commonly diagnosed cancer in the world. The incidence has been rising sharply in some Asian countries, including Japan, Singapore, and Korea, which were previously known as low-risk areas in the last few decades. According to the National Cancer Registry of Korea, the age-standardized incidence rates of total CRC increased by $6.2 \%$ and $6.8 \%$ for men and women, respectively, between 1999 and 2009 [1]. Although the mortality rate from CRC started to decline among younger generations and women, it is still ranked as one of the most common causes of cancerrelated death in Korea [2].

Over the past few decades, significant therapeutic improvements have been made in the treatment of 
colon cancer. However, most patients with advanced colon cancer are treated with fluoropyrimidine-based chemotherapy in combination with or without irinotecan or oxaliplatin [2]. Recently, molecularly targeted drugs have been developed for use against specific cancers and provide additional clinical benefits to patients. These novel drugs, including cetuximab (Erbitux ${ }^{\circledR}$; Merck KGaA, Germany) and bevacizumab (Avastin ${ }^{\circledR}$; Genentech Inc., USA), have been approved for CRC treatment and improve the survival rate by more than 30 $\%$ [3-8]. Nevertheless, there are serious limitations of the targeted drugs to intervene with colon cancer because the molecular characteristics of colon cancer is poorly understood $[9,10]$, and the clinical benefit of targeted therapeutics is still limited $[11,12]$.

Recent advances within cancer genomics identify mutually exclusive oncogenic drivers to trigger a personalized treatment in various cancers. However, insufficient clinical characterization of the drivers often led to the poor response in clinical trials with targeted therapeutics [13-15]. Therefore, it is important to associate the long-term clinical outcome of cancer patients with certain genomic alterations through a systemic approach.

The overall goal of this study was to identify clinically confident targets for the therapeutic intervention of colon cancer. To this end, we performed RNA-seq with tumors from Korean patients with colon cancer. From sequence data, we analyzed gene fusions, differential gene expression, and non-synonymous somatic mutations. Gene fusions were verified by the sequencing of the fusion transcripts, FISH, and immunohistochemistry. The oncogenicity of the fusion genes was validated by an in vitro colony formation assay and an in vivo xenograft study with transformed cell lines with fusion transcripts. The clinical effect of the fusion genes was addressed by analyzing the prevalence and the overall survival of the patients having the genomic alterations from an independent retrospective cohort.

\section{RESULTS}

\section{The identification of NTRK1 fusions among Korean patients with colon cancer}

An RNA-seq cohort was composed of 79 male and 71 female patients with a median age of 60 at diagnosis. The majority of the cancer was located in the ascending colon (48 out of $150 ; 32 \%$ ) or in the sigmoid (73 out of $150 ; 48.7 \%)$. Half of the cancers were determined at stage I (12 out of $150 ; 8 \%$ ) or stage II (63 out of 150 ; $42 \%$ ), and the other half were at stage III ( 75 out of 150 ; $50 \%$ ). Microsatellite analysis revealed that 127 of 150 tumors $(84.7 \%)$ were microsatellite stable (MSS), and 21 of 150 tumors (14\%) had highly unstable microsatellites
(MSI-H). Clinical follow-up demonstrated that 23 of 150 patients $(15.3 \%)$ have experienced disease progression within three years of diagnosis (Table S1). RNA-seq generated a median of 118.5 million mappable reads with a lower base call accuracy of 99\% (Q20) $=94.3 \%$ (Figure S1 and Table S2). Principle component analysis (PCA) with 18,725 expressed genes from individual tumors revealed three outliers (Figure S2 and Table S2); therefore, 147 tumors and 47 matched normal controls were used for gene fusion analysis. We applied GFP [16], defuse [17], and FusionMap [18], and nine in-frame fusions were found based on two out of three algorithms with discordant paired-end reads, as well as a spanning read cutoff $=10$ and a chromosomal distance cutoff $=100$ $\mathrm{Kb}$ when intrachromosomally rearranged. Gene fusion was validated by exon expression analysis of donor and acceptor genes (Table S3). Those included PTPRK-RSPO3 in two patients $(1.4 \%), N A G L U-I K Z F 3, G T F 3 A-C D K 8$, RAD51AP1-AKAP3, RASA1-LOC644100 in each single patient $(0.7 \%)$, and $L M N A$ or TPM3-NTRK1 in three patients $(2 \%)$. We then further investigated NTRK1 fusions because NTRK1, which encodes for membrane-bound TrkA protein, has been shown to be rearranged with TPM3 in colon carcinoma [19] and papillary thyroid carcinoma [20]. MPRIP-NTRK1 and CD74-NTRK1 were found in lung adenocarcinoma [21], TP53-NTRK1 and LMNANTRK1 in Spitzoid neoplasm [22], RABGAP1L-NTRK1 in intrahepatic cholangiocarcinoma [23], and NFASCNTRK1 and BCAN-NTRK1 in glioblastoma multiforme [24]. To our knowledge, LMNA-NTRK1 fusion was not reported in colon cancer. Furthermore, the prevalence and the clinical relevance of NTRK1 fusions remain largely unknown in colon cancer. The exon expression of the NTRK1 gene was exclusively detected in tumors harboring NTRK1 fusions (Figure 1A). Subsequently, we confirmed the exon junctions in the fusion transcript of LMNA-NTRK1 and TPM3-NTRK1 by Sanger sequencing (Figure 1B), and NTRK1-encoded TrkA expression by immunohistochemistry (Figure 1C). NTRK1 gene fusion was confirmed by a FISH assay with split FISH probes on 5'- and 3'-end of NTRK1 gene (Figure 1D). Schematic rearrangement of the NTRK1 gene (Figure 1E) and the architecture of TrkA fusion protein demonstrated that the protein kinase domain of the TrkA protein is well conserved after gene fusion (Figure 1F).

\section{Mutually exclusive oncogenicity of NTRK1 fusions in colon cancer}

Whereas the oncogenicity of NTRK1 fusions was well characterized in papillary thyroid carcinoma [25] and lung adenocarcinoma [21], it remains to be elucidated in colon cancer. To address the oncogenicity of LMNANTRK1 and TPM3-NTRK1 fusions in colon cancer, we conducted in silico, in vitro, and in vivo approaches. 
NTRK1 fusions were mutually exclusive to oncogenic mutations in CRC (Figure 2). Tumors harboring NTRK1 fusions did not have non-synonymous somatic mutations in KRAS, NRAS, PIK3CA and other putative oncogenes. In contrast, somatic mutations in various suppressor genes, including TP53, $A P C$, and $F B X W 7$, were observed in $N T R K 1$ fusion-positive tumors. It is noteworthy that most of the suppressor mutations were found in the NTRK1 fusion-positive tumor with highly unstable microsatellites (Patient ID: LGP088T). We removed potential germline variants, as described in Methods. However, it is likely that unpaired tumor tissues may have more somatic variants than paired tumor tissues. Therefore, the negative selection of somatic variants was preceded and revealed the mutually exclusive oncogenicity of NTRK1 fusions in colon cancer. Although we performed the clinical follow-up of the patients, most of the patients were diagnosed after 2010 and it is too soon to determine any clinicopathological effects of the NTRK1 fusions in colon cancer. In subsequent analyses of the oncogenicity of NTRK1 fusions, we generated $L M N A(\mathrm{e} 6)-N T R K 1(\mathrm{e} 11)$ or TPM3(e8)-NTRK1(e9, e11, e12) fusion transcripts harboring plasmid DNA for cell transformation. TrkA protein from a transformed NIH3T3 cell line with the fusion transcripts was well expressed (Figure 3A). The NIH3T3 cells overexpressing LMNA-NTRK1 (376 \pm 33 colonies) or TPM3-NTRK1 (243 \pm 46 colonies) formed a significantly larger number of colonies than nontransformed cells $(1 \pm 2$ colonies, $p<0.01)$, which was comparable to KM12 (285 \pm 36 colonies) (Figure 3B). We then evaluated the tumorigenicity of NTRK1 fusions by inoculating immunocompromised athymic-mice with the transformed cells. Tumors from the transformed cells were palpable from day 18 of inoculation, and the volumes of the tumors were comparable to KM12 on day 29 of inoculation (Figure 3C). Although the expression level of
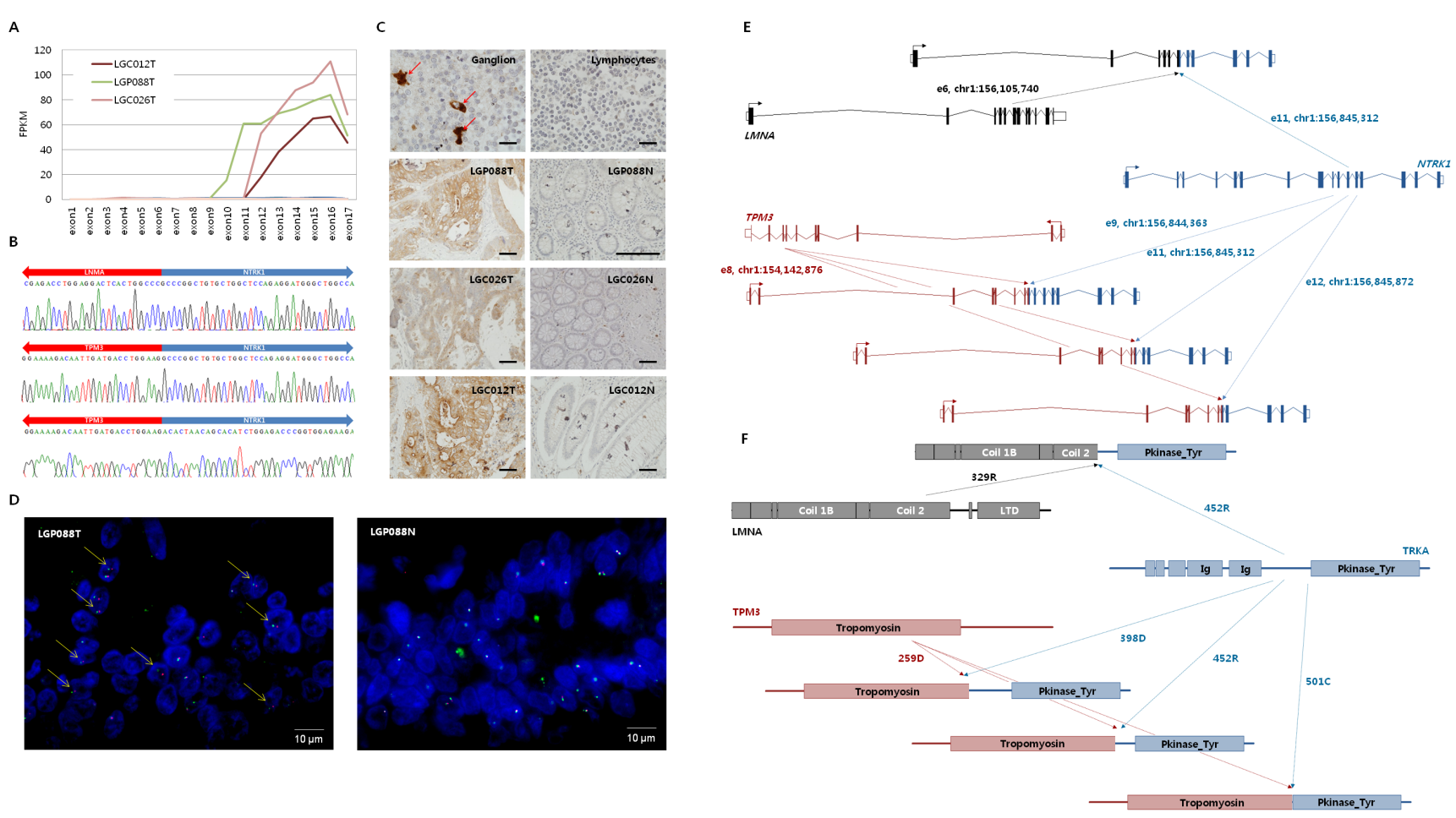

Figure 1: Rearrangement of NTRK1 in colon cancer of Korean patients. A. The exon expression of the NTRK1 gene was exclusively detected in tumors harboring NTRK1 fusions (Sample ID = LGP088T, LGC026T, LGC012T). B. Exon junctions in fusion transcripts of LMNA-NTRK1 and TPM3-NTRK1 were confirmed by RT-PCR followed by Sanger sequencing. The NTRK1 gene at chr1:156,845,312 was rearranged with the LMNA gene at chr1:156,105,740, and the NTRK1 gene at chr1: 156,844,363 or 156,845,312, or 156,845, 872 was rearranged with the TPM3 gene at chr1:154,142,876, respectively. C. Immunohistochemical analysis with antiTrkA protein, C-terminal antibody showed tumor-specific, cytoplasmic expression of TrkA protein in NTRK1 fusion-positive samples. Brain ganglions and lymphocytes served as positive and negative control, respectively. The scale bar $=25 \mu \mathrm{m}$. D. Fluorescence in situ hybridization (FISH) assay with split FISH probes to reassure NTRK1 rearrangement. TexRed-labeled 5'-end NTRK1 probe is located on chr1:156,390 Kb - 156,814 Kb (red), and FITC-labeled 3'-end NTRK1 probe on chr1:156,851 Kb - 157,630 Kb (green). Split red and green signals were observed in a representative NTRK1 fusion-positive tumor tissue (LGP088T), but not in matched normal tissue (LGP088N). Yellow arrows indicate NTRK1 fusion genes. The scale bar indicates $10 \mu \mathrm{m}$. E. Schematic rearrangement of NTRK1 gene. Rearrangement between $L M N A(\mathrm{e} 6)$ and $N T R K 1(\mathrm{e} 11)$ generated a single isoform of the fusion transcript, and rearrangement between TPM3(e8) and $\operatorname{NTRK1}(\mathrm{e} 9, \mathrm{e} 11, \mathrm{e} 12)$ generated three isoforms of the fusion transcripts. F. Architecture of TrkA fusion proteins. The putative structure of NTRK1 fusion genes demonstrated that the protein kinase domain of TrkA protein was well conserved. 
LMNA-TrkA fusion protein in NIH3T3 cells was less than that of the other two fusion protein-expressing cell lines, oncogenic activity was comparable with that of the other fusion proteins. Therefore, these results demonstrated that LMNA-NTRK1 and TPM3-NTRK1 could be mutually exclusive cancer drivers in colon cancer.

\section{Clinical relevance of $N T R K 1$ fusions}

The clinical relevance of NTRK1 fusion was assessed with independent cohorts that were comprised of 216 Korean and 472 Chinese patients with colon cancer. We investigated the prevalence of TrkA protein expression by using tissue microarray (TMA) constructed from the cohorts. Immunohistochemical analyses revealed that TrkA was strongly expressed in the cytoplasm of the tumor cells in 11 Korean (5.1\%) and 28 Chinese patients (5.9\%), respectively, and the frequency of TrkA expression was not significantly different between the two populations $(p=0.1657)$ (Figure 4A). Those frequencies were higher than our expectation from RNA-seq results. To verify the correlation between TrkA protein expression and NTRK1 fusion, 15 tumor tissues with or without TrkA protein expression from Korean patients were subject to FISH analysis. Split FISH signals were significantly detected in TrkA-positive tumors ( $p=0.0192$, Figure $4 \mathrm{C}$ to $4 \mathrm{D}$, Table $\mathrm{S} 4$ ), but not in the TrkA negative tumors (Figure 4E), thus indicating that TrkA protein expression was partly a result of NTRK 1 fusion. Among the 216 Korean patients, 42 patients $(24.1 \%)$ were had died within 10 years after diagnosis. All of the 216 Korean patients were divided into two groups according to the cytoplasmic TrkA expression level. Clinicopathological characterization revealed that the tumors with cytoplasmic TrkA expression were localized in the left colon $(p=0.0504)$ and frequent at the T3 depth of invasion ( $p=0.0437)$. TrkA positive tumors were significantly associated with the occurrence of perineural invasion/lymphovascular emboli $(p=0.0429)$, and most of the tumors were microsatellite stable $(p=$ 0.0024 ) (Table 1). In accordance with the histopathological and molecular status of the tumors, Kaplan-Meier survival analysis demonstrated that the survival time of TrkApositive patients was marginally but significantly shorter than TrkA-negative patients (Figure 5, HR $=0.5346,95 \%$ $\mathrm{CI}=0.2548$ to $0.9722, p=0.0411)$. Taken together, these results imply that NTRK1 fusion could be a clinically relevant target for the therapeutic intervention of colon cancer.

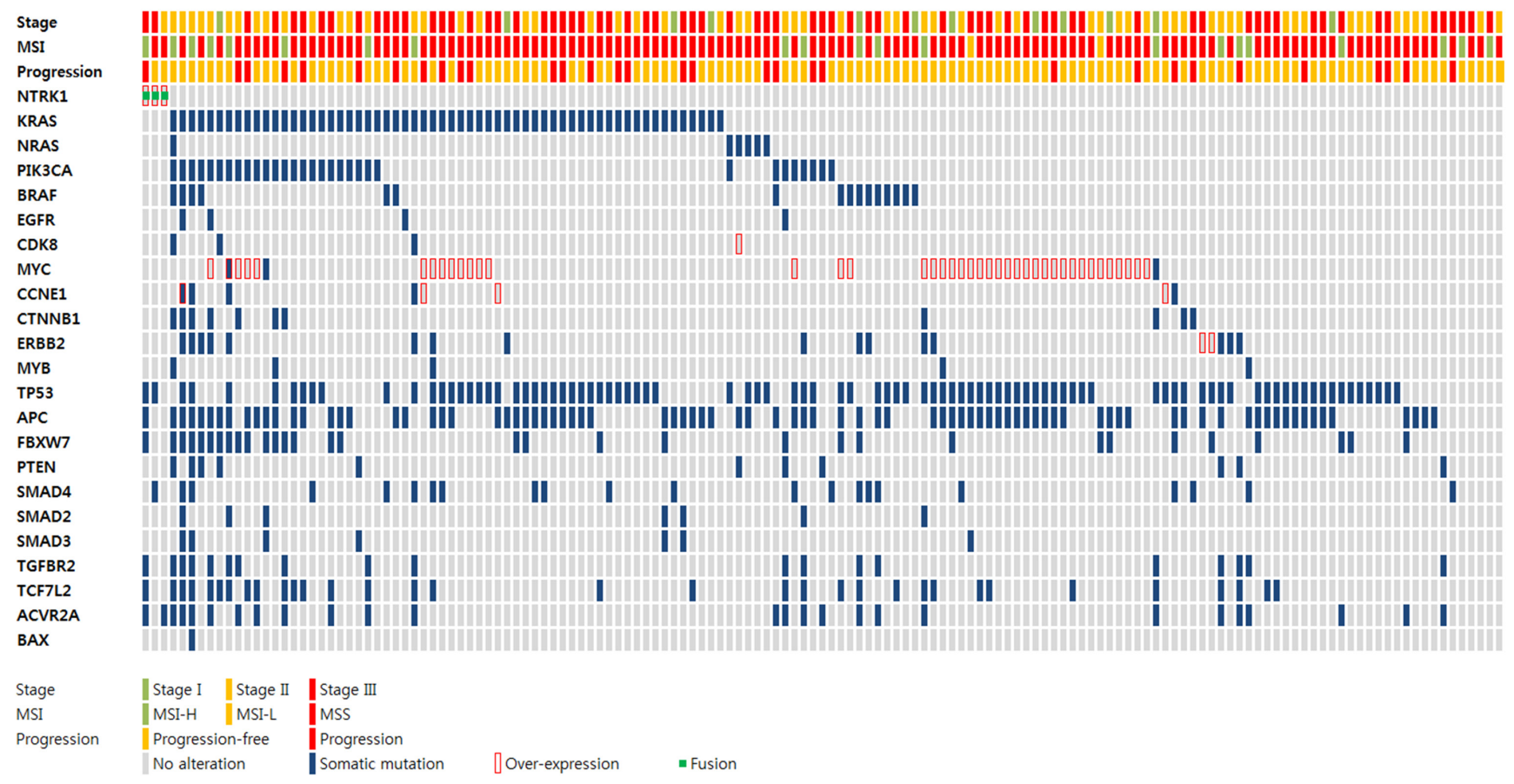

Figure 2: Mutual exclusivity of NTRK1 fusion to oncogenic mutations in colon cancer. The tumor stage was denoted by colored squares (i.e., green for stage I, yellow for stage II, and red for stage III). Microsatellite stability was symbolized as a green square for microsatellite instable-high (MSI-H), a yellow square for microsatellite instable-low (MSI-L), and a red square for microsatellite stable (MSS). Tumor progression was designated by yellow squares (i.e., progression-free) and red (i.e., progression). Any alterations in representative oncogenes and suppressor genes including NTRK1 in colon cancer were depicted as follows: gray squares for no alteration, blue squares for non-synonymous somatic mutation, red squares for over-expression, and green dots for NTRK1 fusion. NTRK1 fusions were mutually exclusive to representative oncogenic mutations in colon cancer. Somatic mutations in various suppressor genes were denoted including TP53, $A P C$, and $F B X W 7$, particularly in the MSI-H sample. 
A

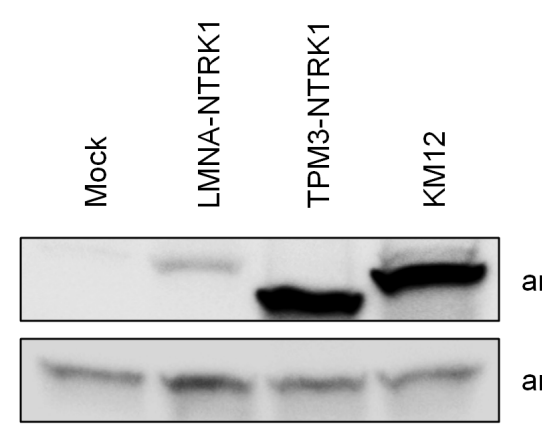

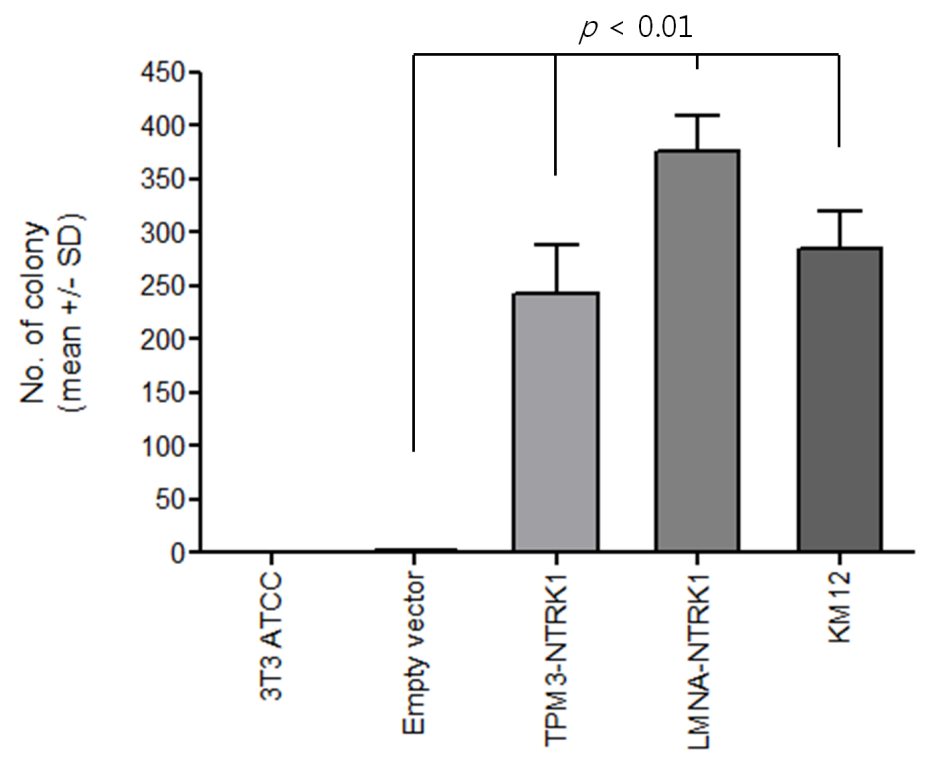

C
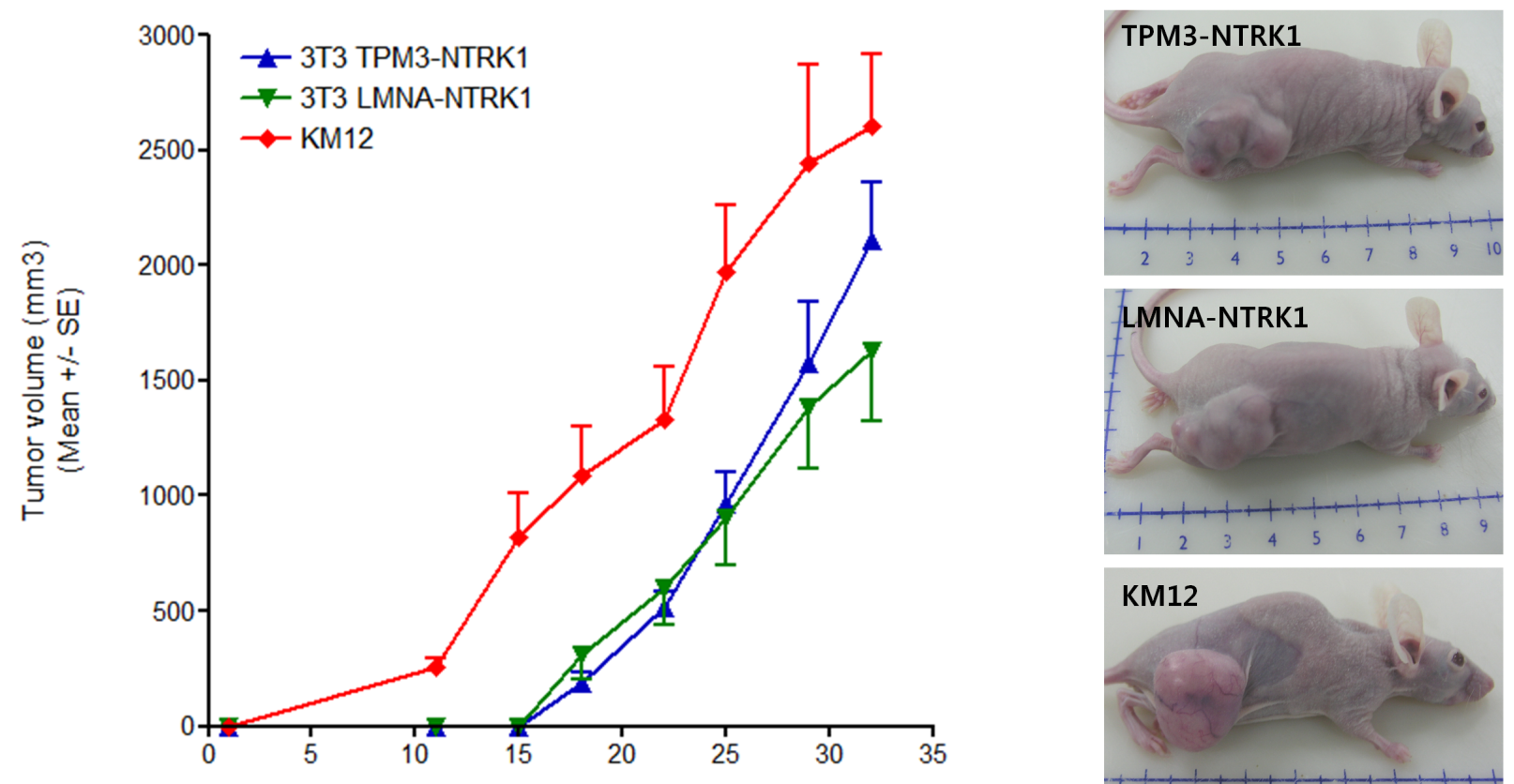

Days of innoculation
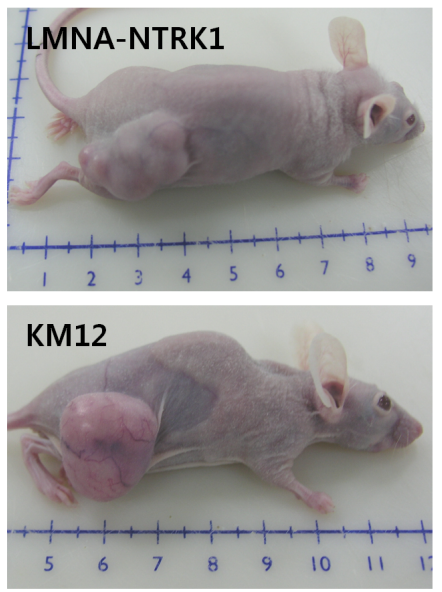

Figure 3: Tumorigenicity assay with transformed NIH3T3 cells. A. TrkA proteins from a transformed NIH3T3 cell line with LMNA-NTRK1 or TPM3-NTRK1 fusion transcript and KM12 were subject to Western blot using the anti-TrkA protein, C-terminal monoclonal antibodies. B. A total of 1,200 cells were seeded in the $0.3 \%$ of top agarose on the $0.5 \%$ of base agarose per well of a $24-$ well plate and colonies were allowed to form for 3 weeks. NIH3T3 cells transduced with exogenous expression of LMNA-NTRK1 or TPM3-NTRK1 formed a small but significantly large number of colonies (376 \pm 33 and $243 \pm 36$ colonies, respectively) in comparison to empty vector control $(1 \pm 2$ colonies, $p<0.01)$, which was comparable to KM12 (285 \pm 36 colonies). A colony formation assay was independently performed in triplicate. The number of colonies was denoted as mean \pm standard deviation. C. NIH3T3 cells transduced with an exogenous expression of LMNA-NTRK1 or TPM3-NTRK1 at 1 $10^{6}$ cells per site were inoculated subcutaneously in the right dorsal region of immunocompromised athymic mice (5 mice per group). When the tumor became palpable, the tumor volume was measured every three days until the thirty-second day after inoculation. Transformed NIH3T3 cell-driven tumors were grown comparable to KM12. Tumor volumes were denoted as a mean \pm standard error. Representative animals with tumors from LMNA-NTRK1 or TPM3-NTRK1 transgene or KM12 were presented. The unit is centimeter. 


\section{Therapeutic intervention of NTRK1 fusion}

Therapeutic intervention of NTRK1 fusion-driven cell growth was evaluated in KM12 cells. Although there are several cell lines harboring $N T R K$ fusion, including CUTO-3 lung cancer cells with MPRIP-NTRK1 fusion and MO-91 acute myeloid leukemia (AML) cells with ETV6-NTRK3 fusion, KM12 is the only available colon cancer cell line harboring NTRK1 fusion. Therefore it

A

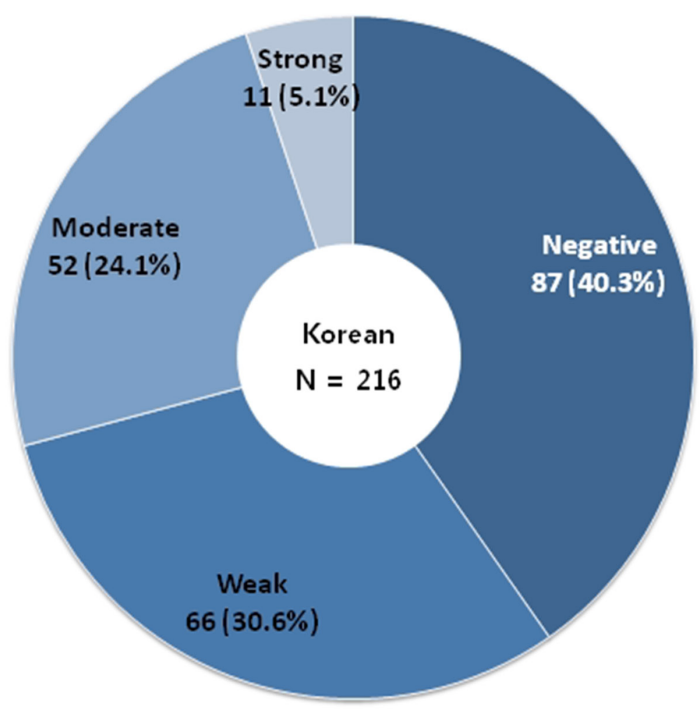

B
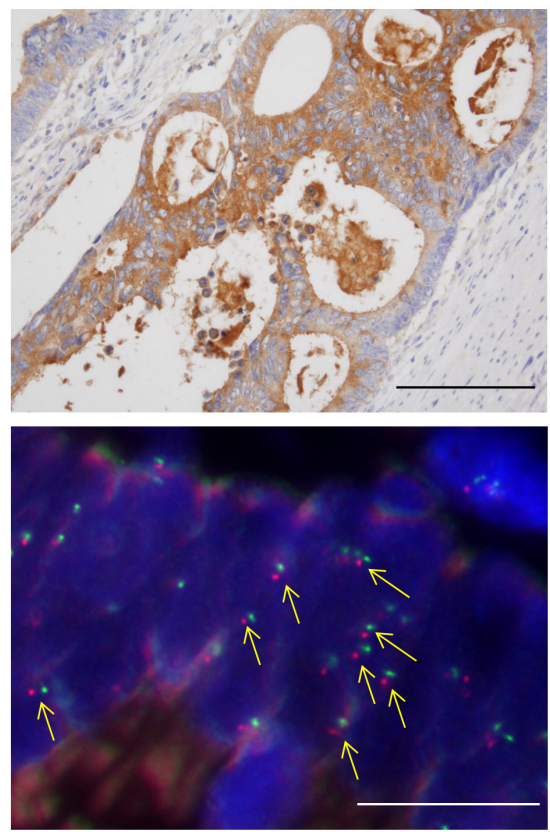

C
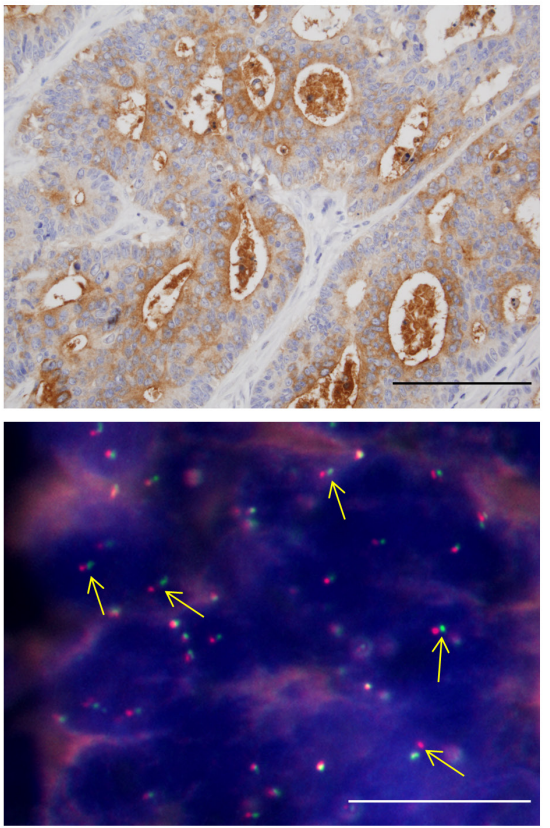

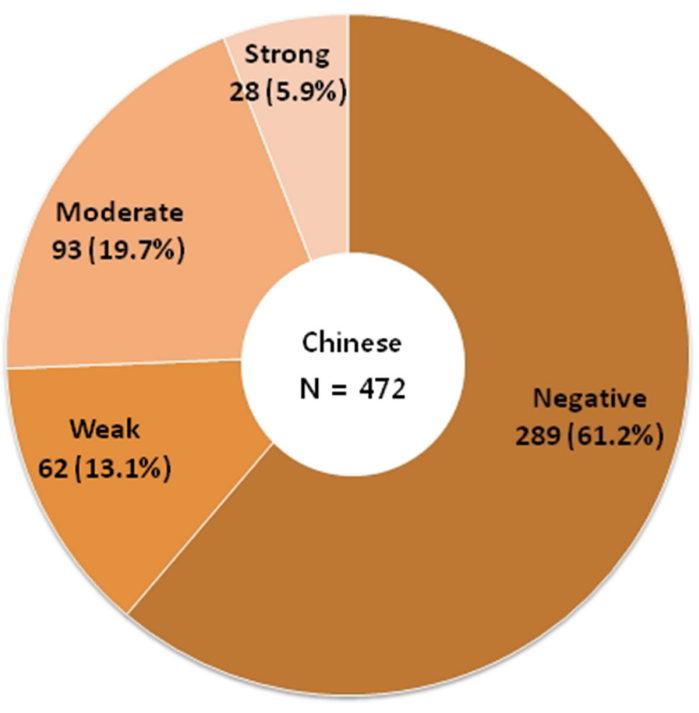

D

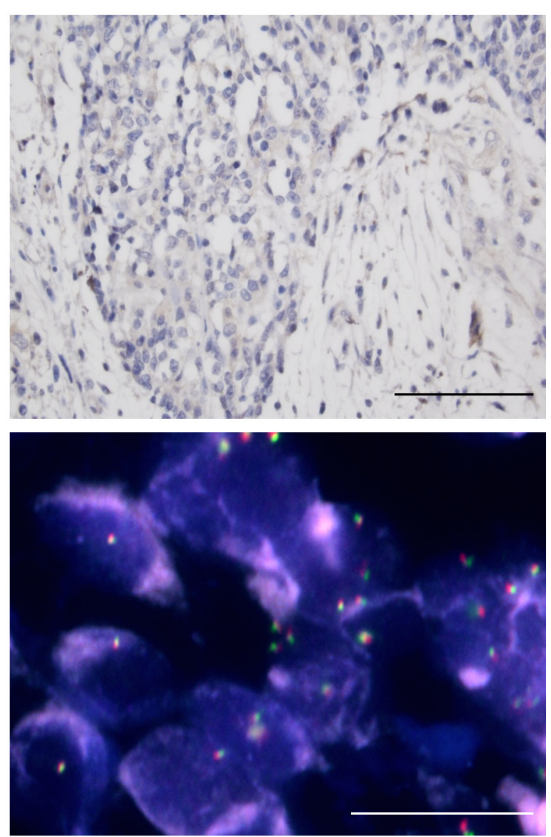

Figure 4: The prevalence of TrkA protein expression in Korean and Chinese patients with colon cancer. Pie graphs depict the proportion of subpopulations with a different degree of TrkA protein expression. A. In Korean patients, 87 out of 216 patients (40.3\%) were TrkA negative, and 66 out of 216 patients (30.6\%) had weak TrkA expression (both subpopulations were determined to be TrkA negative). Fifty-two (24.1\%) and 11 patients (5.1\%) with a moderate to strong expression of TrkA protein were identified and designated as TrkA positive. In Chinese patients, 351 out of 472 patients (74.3\%) were TrkA negative, and 121 out of 472 patients (25.6\%) were TrkA positive. Out of the Chinese population of 472, $289(61.2 \%)$ and 62 patients (13.1\%) were TrkA negative, and 93 (19.7\%) and 28 patients (5.9\%) were TrkA positive. B. Immunohistochemistry represents strong cytoplasmic TrkA expression in a colon cancer (sample ID: Colon_50_FISH01), and FISH analysis confirmed the frequent NTRK1 rearrangement in the tissue. C. Immunohistochemistry represents moderate cytoplasmic TrkA expression in a colon cancer (sample ID: Colon_50_FISH06), and FISH analysis confirmed the less frequent NTRK1 rearrangement in the tissue. D. Immunohistochemistry represents negative cytoplasmic TrkA expression in a colon cancer (sample ID: Colon_50_FISH11), and FISH analysis confirmed that there was no detectable NTRK1 rearrangement in the tissue. Yellow arrows indicate NTRK1 fusion genes. The scale bar indicates $10 \mu \mathrm{m}$. 
is useful to use KM12 for high throughput screening of drug candidates for NTRK1 fusion-positive colon cancer treatment. We determined the $50 \%$ cytotoxic concentration (CC50) of Lestaurtinib, Crizotinib and ARRY-470 on KM12. Lestaurtinib is an indolocarbazole derivative to inhibit several tyrosine kinases, including FLT3 and TrkA. It had been in phase II/III trials for the oral treatment of relapsed AML. Crizotinib, a dual inhibitor of hepatocyte growth factor receptor (c-Met/HGFR) kinase and anaplastic lymphoma kinase (ALK), was approved and launched in the U.S. in August 2011 for the treatment of patients with ALK-positive advanced or metastatic non-small cell lung cancer (NSCLC). ARRY-470 (also known as LOXO-101) is a selective TrkA inhibitor in

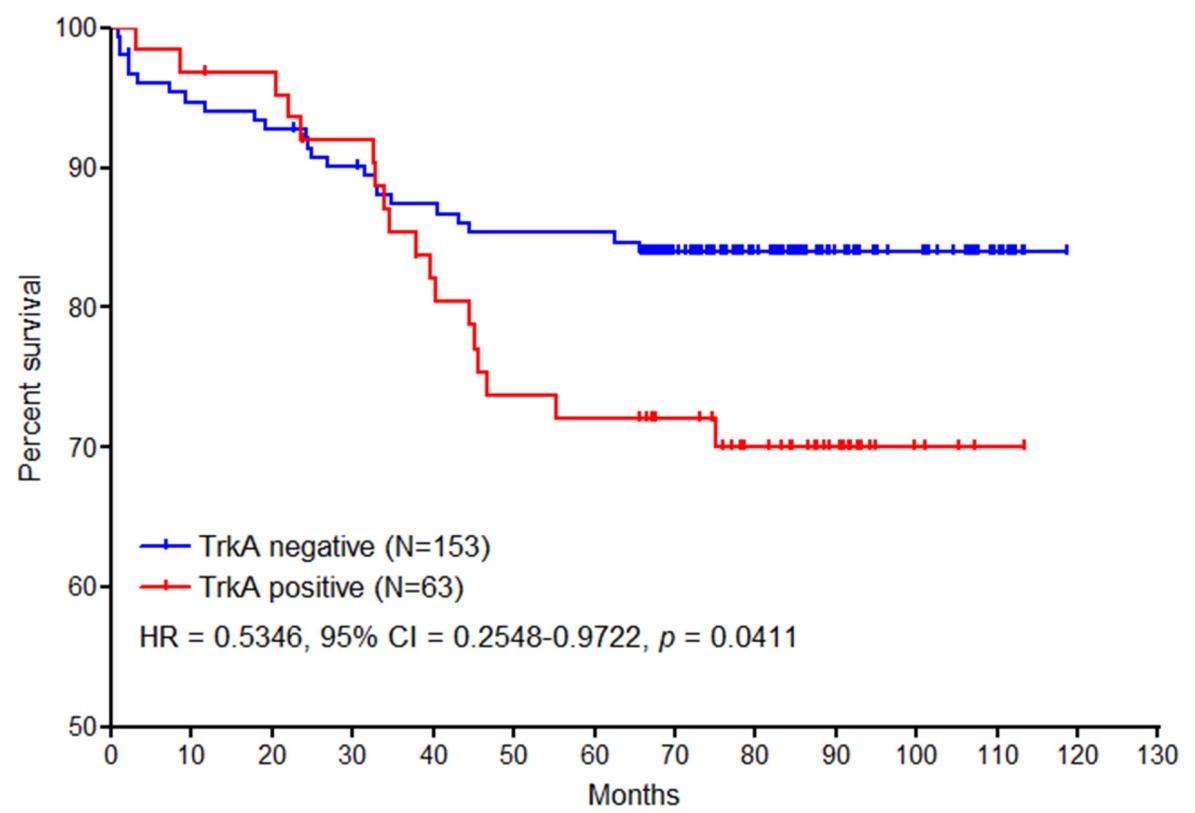

Figure 5: The Kaplan-Meier survival analysis of TrkA-positive and negative patients with colon cancer. Out of 216 Korean patients with colon cancer, 153 patients were determined to be TrkA negative, and 63 patients were TrkA positive, respectively. Patient survival was followed up to 118.6 months. The Kaplan-Meier survival analysis demonstrated that the survival time of cytoplasmic TrkA-positive patients was significantly shorter than TrkA-negative patients with T1-3 colon cancer $(p=0.0411, \mathrm{HR}=0.5346,95 \% \mathrm{CI}=$ 0.2548 to 0.9722 ).
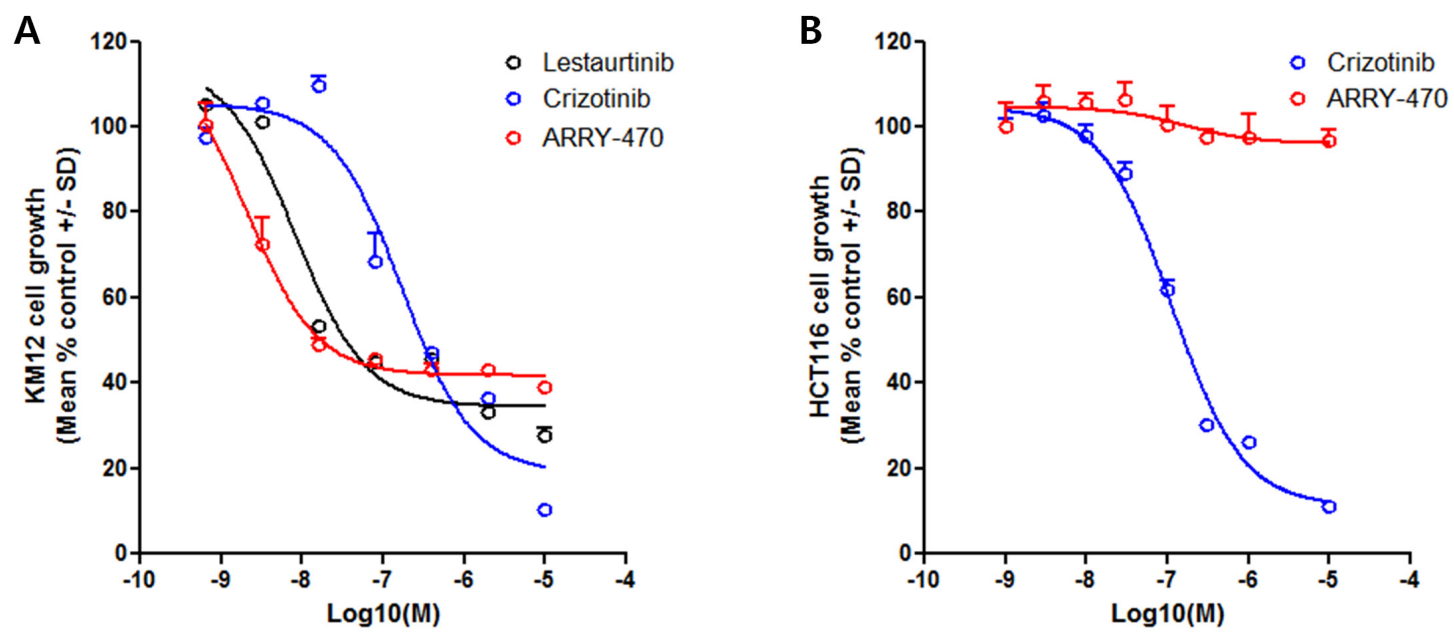

Figure 6: Therapeutic intervention of $N T R K 1$ fusion-driven cell growth. A. Therapeutic intervention of NTRK1 fusion-positive colon cancer cells. Therapeutic intervention of NTRK1 fusion-driven cell growth was evaluated in KM12 cells with Lestaurtinib, Crizotinib, and ARRY-470. KM12 was treated at $0.64 \mathrm{nM}$ to $10 \mu \mathrm{M}$ of each compounds for 4 days, and cell growth was evaluated by ATP-Glo Bioluminometric Cell Viability Assay kit (Biotium Inc.). KM12 was sensitive to Lestaurtinib and ARRY-470 with $10.7 \mathrm{nM}$ and $3.2 \mathrm{nM}$ of CC50, respectively. Crizotinib was less potent to inhibit KM12 proliferation with CC50 $=184.8 \mathrm{nM}$. B. Therapeutic intervention of NTRK1 fusion-negative colon cancer cells. HCT116 was resistant to ARRY-470. Crizotinib was more potent to inhibit HCT116 proliferation with $\mathrm{CC} 50=568 \mathrm{nM}$. 
Table 1: Clinicopathological characteristics of the cohort of 216 Korean patients

\begin{tabular}{|c|c|c|c|c|}
\hline & \multirow{2}{*}{$\mathbf{N}$} & \multicolumn{2}{|c|}{ Cytoplasmic TrkA } & \multirow{2}{*}{$P$ value } \\
\hline & & $(-)$ & $(+)$ & \\
\hline Age (years) & 216 & 70.35 & 70.22 & 0.4675 \\
\hline Size $(\mathrm{cm})$ & 216 & 5.199 & 5.548 & 0.2959 \\
\hline Sex & & & & 0.3351 \\
\hline Male & 118 & 85 & 33 & \\
\hline Female & 98 & 68 & 30 & \\
\hline Location & & & & 0.0504 \\
\hline Right colon & 87 & 67 & 20 & \\
\hline Left colon & 129 & 86 & 43 & \\
\hline Histological type & & & & 0.0761 \\
\hline Well & 16 & 13 & 3 & \\
\hline Moderate & 178 & 120 & 58 & \\
\hline Poor & 10 & 10 & 0 & \\
\hline Mucinous & 12 & 10 & 2 & \\
\hline Invasion depth (T1-2 vs. T3) & & & & 0.0437 \\
\hline $\mathrm{T} 1$ & 12 & 10 & 2 & \\
\hline $\mathrm{T} 2$ & 23 & 19 & 4 & \\
\hline T3 & 181 & 124 & 57 & \\
\hline Perineural invasion (PNI) & & & & 0.1315 \\
\hline Negative & 149 & 109 & 40 & \\
\hline Positive & 67 & 44 & 23 & \\
\hline Lymphovascular emboli (LVE) & & & & 0.2229 \\
\hline Negative & 142 & 103 & 39 & \\
\hline Positive & 74 & 50 & 24 & \\
\hline $\mathrm{PNI} / \mathrm{LVE}$ & & & & 0.0429 \\
\hline Negative & 119 & 90 & 29 & \\
\hline Positive & 97 & 63 & 34 & \\
\hline Lymphnode metastasis (N0 vs N1-2) & & & & 0.015 \\
\hline N0 & 124 & 95 & 29 & \\
\hline N1a & 40 & 23 & 17 & \\
\hline N1b & 32 & 22 & 10 & \\
\hline $\mathrm{N} 2 \mathrm{a}$ & 13 & 9 & 4 & \\
\hline $\mathrm{N} 2 \mathrm{~b}$ & 7 & 4 & 3 & \\
\hline Microsatellite status & & & & 0.0024 \\
\hline MSS & 180 & 119 & 61 & \\
\hline MSI-L & 6 & 5 & 1 & \\
\hline MSI-H & 30 & 29 & 1 & \\
\hline
\end{tabular}

phase I clinical studies for the oral treatment of solid tumors. In 2015, orphan drug designation was assigned to the compound in the U.S. for the treatment of soft tissue sarcoma (https://integrity.thomson-pharma.com). In consistent with the previous study [21], KM12 was sensitive to ARRY-470 $(\mathrm{CC} 50=3.2 \mathrm{nM})$ and Lestaurtinib $(\mathrm{CC} 50=10.7 \mathrm{nM})$. Crizotinib was less potent to inhibit KM12 proliferation with CC50 $=184.8 \mathrm{nM}$ (Figure 6A). This modest activity of Crizotinib could be due to nonTrkA kinase effects. In contrast, ARRY-470 had a poor inhibitory effect on HCT116 which has a mutation in codon 13 of the RAS proto-oncogene without NTRK1 fusion. Crizotinib was more potent to inhibit HCT116 proliferation as expected (Figure 6B). Taken together, these results demonstrated that the use of TrkA kinasespecific inhibitors may provide a new therapeutic strategy for targeted treatment not only for NTRK1 fusion-driven lung adenocarcinoma and sarcoma but also for colon cancer.

\section{DISCUSSION}

In this study, we applied RNA-seq to identify target candidates for therapeutic intervention of colon cancer. 
Gene fusion is one of the most important oncogenic mechanisms due to uncontrolled protein expression through the rearrangement of ubiquitously expressed donor genes and acceptor oncogenes. RNA-Seq is advantageous for the discovery of fusion genes compared to the whole genome sequencing because it can identify the fusion genes that are specifically translated into proteins. Therefore, RNA-seq has been widely used to identify active fusion genes. Our results confirm that RNA-seq is an efficient tool to find fusion genes to drive colon cancer. Especially, 3'-NTRK1 transcripts were expressed only in tumors harboring LMNA-NTRK1 or TPM3-NTRK1 fusion genes, and they were precisely concomitant with protein expression (Figure 1). In the previous studies, TCGA did not find NTRK1 fusion in CRC [9] and Genentech reported a single TPM3-NTRK1 fusion out of 72 colon tumors $(1.4 \%)$ [10]. In the current study, we found one LMNA-NTRK1 and two TPM3-NTRK1 fusions among 147 Korean patients with colon cancer. The frequency of NTRK1 fusion was a little higher than in the previous studies $[10,26]$, which could possibly be explained by high pass paired-end transcriptome sequencing that we applied. In addition, there could be ethnic differences in the frequency of the fusion because we investigated the Korean population only in the current study. In addition to NTRK1 fusions, we found two PTPRK-RSPO3 fusions, and each of NAGLU-IKZF3, GTF3A-CDK8, RAD51AP1$A K A P 3$, and RASA1-LOC644100 fusion (Table S3). Recurrent RSPO fusions have been known to occur in $10 \%$ of colon tumors. $R S P O$ fusions were mutually exclusive with $A P C$ mutations and capable of potentiating Wnt signaling [10]. The other fusions have not been reported yet in various tumors, and their oncogenic potential remains to be clarified. Therefore, with the development of sequencing capability and bioinformatics analytics tools, RNA-seq will eventually be an indispensable tool in drug discovery and development.

Most genomic studies on cancer to date use tumor tissues from a prospective collection $[9,10]$. Many studies have reported on the clinicopathological characteristics of tumors harboring certain genomic alterations; however, a lack of sufficient follow-up information makes obscure the clinical relevance of certain genomic alterations. Some tumors do not respond to treatments against the genomics-based and preclinically-proven driver oncogenic alterations, and insufficient clinicopathological characterization of the cancer drivers may be the reason for the failure of investigational new targeted drugs in clinical trials. For instance, RET fusions were recognized as oncogenic and drug-sensitive rearrangements in approximately $1-2 \%$ of lung adenocarcinoma $[16,27]$ and various multi-kinase inhibitors were actively investigated to treat non-small cell lung cancer. However, the clinical significance of RET and/or RET fusion genes is not fully understood, which led to the failure to prove the clinical benefit of vandetanib [28], sorafenib [29], and erlotinib
[30]. The previous studies support how important the confirmation of the clinicopathological relevance of certain genomic alterations is to provide clinically confident therapeutic targets. Therefore, to obtain clinically relevant therapeutic targets, not only genomics or preclinical evidence of oncogenicity, but also long-term follow-up would be useful. We expect that the current clinical evaluation, accompanied with a genomic analysis of cancer, would accelerate the translational transition of genomic research in cancer and find clinically confident therapeutic targets from cancer genomics.

In this study, we observed that cytoplasmic TrkA was strongly detected in 11 out of 216 Korean patients with colon cancer $(5.1 \%)$, and it was comparable to the prevalence in Chinese patients (Figure 4). The incidence of cytoplasmic TrkA was higher than that of RNA-seq. We verified the immunohistochemical results with FISH, and it turned out that NTRK1 fusion was significantly correlated with the expression level of TrkA protein. Therefore, it appears that NTRK1 fusion could be a possible cause of TrkA overexpression. We cannot exclude the fact that different patient cohorts for RNA-seq and TMA could contribute this discrepancy. Different molecular stability of NTRK1 mRNA and TrkA protein, and different sensitivity of detection methods by RNA-seq or immunohistochemistry may contribute the discrepancy as well. Cytoplasmic staining in the remainder of the specimen might represent undetected NTRK 1 fusions. Since we applied FISH on tissue slides, probe penetration would be limited compared to cell or metaphase chromosomes. Although RNA-seq is a very sensitive genomic tool to identify fusion gene, it depends on target RNA stability. Therefore, we anticipate that immunohistochemistry accompanied with FISH could be used for the prognosis of colon cancer-harboring NTRK1 fusion.

TrkA expression was higher in T3 stage tumors than T1 and T2 tumors $(p=0.0437)$. With respect to lymph node metastasis, TrkA expression was elevated in N1 and $\mathrm{N} 2$ stage tumors relative to N0 stage tumor $(p=0.015)$. In addition, the frequency of TrkA overexpression was higher in microsatellite-stable tumors than microsatellite-unstable tumors $(p=0.0024)$. In the survival analysis, high TrkA expression was significantly associated with poorer overall survival in colon cancer patients (Figure 5). These results demonstrate that NTRK1 fusion, as well as NTRK1 fusionderived TrkA overexpression, would provide information on the overall survival of the patients with colon cancer. Furthermore, these alterations would give information on the effect of a therapeutic intervention. It suggests that NTRK1 fusion has the potential prognostic and predictive significance in colon cancer.

Conclusively, we identified LMNA-NTRK1 and TPM3-NTRK1 fusion genes in Korean patients with colon cancer through RNA-seq. NTRK1 fusions were mutually exclusive colon cancer drivers with tumorigenicity in cells 
and in animals. Clinicopathological analysis demonstrated that the proportion of patients with NTRK1 fusion-driven TrkA expression was substantial in Korean and Chinese patients with colon cancer, accompanied with poor overall survival. Therefore, NTRK1 fusion was granted as a therapeutic target to treat colon cancer.

\section{MATERIALS AND METHODS}

\section{Guideline compliance}

All of the methods that are described in this study were carried out in accordance with the approved guidelines for the use of experimental animals and human subjects.

\section{Patients}

This study was approved by the institutional review boards (IRBs) of Chonnam National University Hwasun Hospital and Pusan National University Hospital (PNUH), Korea. Fresh frozen tissues resected between 2008 and 2012 from patients with primary colon cancer and matched normal controls were used in this study. Informed consent was obtained from all patients. Tumor tissues were selected according to the tumor sample inclusion criteria of the International Cancer Genome Consortium (ICGC). Briefly, tumor tissues composed of more than $60 \%$ of tumor cells and less than $20 \%$ of necrotic cells or normal cells on histological assessment were included. Tumor tissues from patients who had a family history of colon cancer were excluded. In total, 150 tumor and 50 matched normal tissues were analyzed.

\section{MSI analysis}

Tumor DNA and matched normal DNA from formalin-fixed and paraffin-embedded tissues were used for MSI assessment using Bethesda markers (BAT26, D5S346, BAT25, D17S250, D2S123). DNAs were extracted by using the QIAmp DNA FFPE Tissue Kit (Qiagen, Germany). DNA purity and concentration were measured by the ND-1000 spectrophotometer (Nanodrop technology, USA). Multiplex PCR was performed and MSI was determined by amplicon size in consideration of signal intensity. In case of the failure of MSI analysis due to the poor quality of DNA, microsatellite status was predicted based on total number of somatic mutations including DNA polymerase epsilon (POLE) alterations.

\section{RNA-seq}

Total RNA was extracted by using the RNAiso Plus (Takara Bio, Japan). Extracted RNA was purified by using the RNeasy Mini Kit accompanied with the DNase I (Qiagen) treatment. RNA integrity was assessed on the Bioanalyzer (Agilent, USA), and tumor RNAs with RNA integrity number (RIN) $\geq 6$ were subject to RNAseq (Table S1). RNA-seq libraries were generated by using the TruSeq RNA sample Preparation Kit (Illumina, USA). Briefly, mRNA was enriched by using poly-T oligo-attached magnetic beads, followed by mRNA fragmentation by acoustic shearing. First-strand cDNA was synthesized by using reverse transcriptase and random hexamers, and second-strand cDNA by using DNA polymerase I and RNase H. cDNA was subject to adapter ligation, and then enriched with PCR to prepare cDNA library. cDNA libraries were sequenced on HiSeq 2000 (Illumina) to obtain around 100 million paired-end reads $(2 \times 101 \mathrm{bp})$.

\section{Sequence data analysis}

Sequencing reads from cDNA were aligned with the NCBI human reference genome (hg19) by using GSNAP [31] and TopHat [32] with a 5\% mismatch allowance. To minimize mRNA splicing-caused misalignment, sequencing reads were also aligned to a custom human reference cDNA consisting of 161,250 mRNA sequences obtained from public databases (36,742 RefSeq, 73,671 UCSC, and 161,214 Ensembl) [33].

\section{Gene fusion analysis}

In-frame fusion genes were identified by using GFP [16], and cross validated with defuse [17] and FusionMap [18]. In GFP, putative fusion genes were identified by using discordant read pairs on different genes and exon-spanning reads on the exonic fusion breakpoint of chimeric transcripts, followed by serial filtrations in consideration of strand orientation, sequence homology (E-value $<0.01$ ), spurious reads (spanning $<10 \mathrm{bp}$ ), and spanning read pattern to remove false positives. deFuse and FusionMap were applied to find the actual location of ambiguously aligned spanning reads with a computed split-read analysis which harbored the fusion breakpoint. The fusion gene was determined when it appears to be at least two different algorithms with discordant paired-end reads and a spanning read cutoff $=10$ and a chromosomal distance cutoff $=100 \mathrm{~Kb}$ when intrachromosomally rearranged and out-frame fusions were discarded. It follows read depth assessment in each exon after the fusion breakpoint and determines whether they have been abruptly overexpressed [34]. 


\section{Differentially expressed gene (DEG) analysis}

If not mentioned separately, DEG analysis was performed by using GenePattern at Broad Institute [35]. TopHat alignment was processed using publically available Cufflinks [36] to assemble the reads into transcripts. The number of reads aligned to each gene was normalized by the fragments per kilobase of exon per million (FPKM) [36]. We performed PCA [37] by using the genes with FPKM $>0$ in more than $80 \%$ of samples and outlier samples that did not adhere to the position of either tumor or normal were excluded in further analyses. It resulted in a total of 18,725 genes from 147 tumor and 47 matched normal tissues. Differential gene expression was computed by a pairwise 2 -sided t-test $(p<0.05)$ followed by Benjamini-Hochberg multiple comparison correction [false discovery rate $(\mathrm{FDR})<0.05$ ]. DEGs were determined when the relative expression of genes in tumors was at least 8 times higher than in normal control.

\section{Non-synonymous somatic mutation analysis}

Single nucleotide variances (SNVs) were identified after GSNAP alignment on the custom human reference cDNA. SNVs were defined according to the following criteria: (1) the number of uniquely mapped reads at the position $\geq 2$, (2) the average base quality for the position $\geq 20$, (3) the allele ratio at the position $\geq 3 \%$, (4) the read depth at the position $\geq 10$. Gene annotation was done using RefSeq genes. Potential germline variants were removed by using dbSNP137 at the minor allele frequency $>1 \%$ of samples [38], variants in 59 normal Korean individuals [33], and variants in 47 normal tissue counterparts. However, it is likely that unpaired tumor tissues might have more somatic variants than those of paired tumor tissues; therefore, the negative selection of somatic variants proceeded.

\section{RT-PCR and sanger sequencing}

NTRK1 fusion transcripts were validated with RTPCR from cDNA by using the following primers and conditions: LMNA(e6)-NTRK1(e11) fusion transcripts were amplified at 30 cycles of $30 \mathrm{sec}$ at $94^{\circ} \mathrm{C}, 30 \mathrm{sec}$ at $54^{\circ} \mathrm{C}$, and $30 \mathrm{sec}$ at $72^{\circ} \mathrm{C}$ with forward primer $5^{\prime}-\mathrm{CCA}$ GGT GGA GCA GTA TAA GAA G-3', reverse primer 5'-TGT GGG TTC TCG ATG ATG TG-3' for 354-bp product, and TPM3(e8)-NTRK1(e9, e11, e12) at 30 cycles of $30 \mathrm{sec}$ at $94^{\circ} \mathrm{C}, 30 \mathrm{sec}$ at $55.7^{\circ} \mathrm{C}$, and $30 \mathrm{sec}$ at $72^{\circ} \mathrm{C}$ with forward primer 5'-AAG AAG ATA AAT ATG AGG3', reverse primer 5'-CCG TGC CGC ATA TAC TCA AA3 ' for 406, 553, or 712-bp products, respectively. Eluted PCR products were inserted into TOPO TA vector (Life Technologies, USA), and subjected to Sanger sequencing.
FISH

Commercially available split FISH probes were used to detect NTRK1 fusion according to the manufacturer's guideline (Abnova, Taiwan). Briefly, a deparaffinized and protease-treated formalin-fixed paraffin-embedded (FFPE) tissue section was denatured at $75^{\circ} \mathrm{C}$ and then incubated with the TexRed-labeled probe on the $5^{\prime}$ end and the FITC-labeled probe on 3' end of NTRK1 overnight. After washing and DAPI counterstaining (Abbott, USA), the number and localization of the hybridization signals were assessed. Tumors were determined to be NTRK1 fusion positive when more than 15 out of 100 nuclei demonstrated break-apart 5'- and 3'-end signals.

\section{Immunohistochemistry and clinicopathological characterization}

TrkA expression was confirmed on archival FFPE tumor tissues and matched normal tissues with anti-TrkA protein, C-terminal monoclonal antibody (OriGene, USA). Brain ganglions and lymphocytes served as positive and negative control, respectively. The prevalence of TrkA protein expression was evaluated by using TMA constructed from 216 Korean patients (PNUH cohort) and 472 Chinese patients with colon cancer (Biomax, USA). HE-stained sections from each block were made to define representative tumor regions. TMAs were comprised of two cores of $2 \mathrm{~mm}$ each, and TMA blocks were used for immunostaining. TrkA immunostaining was scored semiquantitatively as follows: negative (-), weak (+), moderate $(++)$, strong $(+++)$. Moderate-to-strong signal was determined to be TrkA positive.

\section{Exogenous expression of the NTRK1 fusion gene in NIH3T3 cells}

Plasmids containing genes of interest were purchased from Origene (USA). LMNA(e6)-NTRK1(e11) and TPM3(e8)-NTRK1(e9, e11, e12) fusion transcripts were generated by the Overlap Extension PCR. Once PCR products containing the fusion transcripts were generated, they were cloned into the pcDNA3.1 (Life Technologies, USA) harboring internal ribosome entry site (IRES)-GFP gene using the Cold Fusion Cloning Kit (System Biosciences, USA), and confirmed by Sanger sequencing. NIH3T3 cells were purchased from the Korean Cell Line Bank (KCLB, Korea) and maintained in glutamine-containing DMEM medium supplemented with $10 \%$ heat inactivated New Born Calf Serum, penicillin and streptomycin. All reagents were purchased from Life Technologies if not mentioned separately. The fusion gene expression vector or empty vector was transduced into the NIH3T3 cells by using the FUGENE 6 (Roche, Germany). 
Cells were selected for at least 20 days by using $800 \mu \mathrm{g} /$ $\mathrm{mL}$ of G418 after transduction, and GFP expressing cells were sorted by BD FACSAriaTM II (BD Biosciences, USA). The exogenous protein expression was tested by Western blotting. Cells were lysed with the RIPA buffer (50 mM Tris pH 7.5, $150 \mathrm{mM} \mathrm{NaCl}, 1 \%$ NP-40, $0.1 \%$ SDS, $50 \mathrm{mM} \mathrm{NaF}, 1 \mathrm{mM} \mathrm{NaVO}, 1 \mathrm{mM}$ EDTA, $1 \%$ Sodium deoxycholate) and applied to the SDS-PAGE. Anti-TrkA antibody was purchased from Abbiotech (USA) and anti- $\alpha$-tubulin was obtained from Cell Signaling Technologies (USA).

\section{Colony formation and xenograft assay}

The clonogenicity and tumorigenicity of NIH3T3 cells with and exogenous expression of $L M N A(\mathrm{e} 6)$ NTRK1(e11) or TPM3(e8)-NTRK1(e9) was evaluated. A total of 1,200 cells were seeded in $0.3 \%$ of top agarose on $0.5 \%$ of base agarose per well of a 24 -well plate, and colonies were allowed to form for 3 weeks. NIH3T3 cells transduced with empty vector and KM12 (KCLB) were used for negative and positive controls, respectively. Colonies were stained with $0.05 \%$ crystal violet and counted under stereomicroscopy. A colony formation assay was independently performed in triplicate. An in vivo tumorigenicity study was approved by the Institutional Animal Care and Use Committee (IACUC) at LG Life Sciences, R\&D Park, Korea. Immunocompromised athymic female Balb/c mice were purchased from Oriental Bio (Korea) at 6 weeks of age. After 1-week of acclimation, the animals were randomly allocated into groups based on bodyweight and health condition. Transformed NIH3T3 cells or KM12 cells at $1 \times 10^{6}$ cells per site were inoculated subcutaneously in the right dorsal region of immunocompromised nude mice (5 mice per group). When the tumor became palpable, the tumor volume was measured every three days until day 32 after inoculation.

\section{Drug screening}

Lestaurtinib and Crizotinib were purchased from Tocris Bioscience (Bristol, UK). ARRY-470 was supplied by LG Life Sciences. KM12 and HCT116 (KCLB) were treated at $0.64 \mathrm{nM}$ to $10 \mu \mathrm{M}$ of each compounds for 4 days, and cell growth was evaluated by the ATP-Glo Bioluminometric Cell Viability Assay Kit (Biotium Inc., USA).

\section{Statistical analyses}

Parametric data were tested for equal variance by applying Bartlett's test. When not significant, data were subject to one-way ANOVA followed by post-hoc
Dunnett's multiple comparison test $(p<0.05)$. Categorical data were subject to a chi-square test $(p<0.05)$.

\section{ACKNOWLEDGMENTS}

The biospecimens for this study were provided by the Pusan National University Hospital and the Chonnam National University Hwasun Hospital or by the members of the National Biobank of Korea, which is supported by the Ministry of Health, Welfare, and Family Affairs. D.Y.P., C.C., and J.P. conceived of the research idea. J.P. designed experiments, took responsibility for the oversight of the project, and wrote the manuscript. H.J., H.R.K., H.S.K., and N.O. collected the biospecimens and clinical data. D.Y.P., C.C., J.H.L., and J.S.L. performed the histopathological examinations and collected the clinical data. J.Y.S. and J.I.K. designed and performed the RNA-seq. E.S. and J.Y.S. performed bioinformatic analyses and contributed to writing of the manuscript. C.H.K. performed the FISH experiments. O.K.P. generated the NIH3T3 cells expressing exogenous NTRK1 fusion genes, drug screening, and contributed to writing of the manuscript. J.P. performed the in vitro clonogenicity experiment. E.P. performed the in vivo tumorigenicity experiment. J.P. (co-author) performed the Western blotting and contributed to the manuscript. H.D.P. and J.S.S. contributed to interpretation of the data.

\section{CONFLICTS OF INTEREST}

D.Y.P., C.C., E.S., J.H.L., C.H.K., O.K.P. and J.P. are listed on a regularized patent application filed with the Korean Intellectual Property Office related to detection and use of NTRK1 fusion in cancer.

\section{GRANT SUPPORT}

This work has been supported in part by a grant (No. 2014R1A2A1A11052217) from the National Research Foundation, the Ministry of Science, ICT and Future Planning, Republic of Korea (D.Y.P.).

\section{Data access}

Sequencing and genotype data has been deposited at the European Genome-Phenome Archive (http://www.ebi. ac.uk/ega/) under accession number EGAS00001001027.

\section{REFERENCES}

1. Shin A, Kim KZ, Jung KW, Park S, Won YJ, Kim J, Kim DY and Oh JH. Increasing trend of colorectal cancer incidence in Korea, 1999-2009. Cancer research and treatment. 2012; 44:219-226.

2. Jung KW, Park S, Won YJ, Kong HJ, Lee JY, Seo HG and 
Lee JS. Prediction of cancer incidence and mortality in Korea, 2012. Cancer research and treatment. 2012; 44:2531.

3. Sobrero AF, Maurel J, Fehrenbacher L, Scheithauer W, Abubakr YA, Lutz MP, Vega-Villegas ME, Eng C, Steinhauer EU, Prausova J, Lenz HJ, Borg C, Middleton G, et al. EPIC: phase III trial of cetuximab plus irinotecan after fluoropyrimidine and oxaliplatin failure in patients with metastatic colorectal cancer. Journal of clinical oncology. 2008; 26:2311-2319.

4. Cunningham D, Humblet Y, Siena S, Khayat D, Bleiberg H, Santoro A, Bets D, Mueser M, Harstrick A, Verslype C, Chau I and Van Cutsem E. Cetuximab monotherapy and cetuximab plus irinotecan in irinotecan-refractory metastatic colorectal cancer. The New England journal of medicine. 2004; 351:337-345.

5. Giantonio BJ, Catalano PJ, Meropol NJ, O'Dwyer PJ, Mitchell EP, Alberts SR, Schwartz MA, Benson AB, 3rd and Eastern Cooperative Oncology Group Study E. Bevacizumab in combination with oxaliplatin, fluorouracil, and leucovorin (FOLFOX4) for previously treated metastatic colorectal cancer: results from the Eastern Cooperative Oncology Group Study E3200. Journal of clinical oncology. 2007; 25:1539-1544.

6. Hurwitz H, Fehrenbacher L, Novotny W, Cartwright T, Hainsworth J, Heim W, Berlin J, Baron A, Griffing S, Holmgren E, Ferrara N, Fyfe G, Rogers B, et al. Bevacizumab plus irinotecan, fluorouracil, and leucovorin for metastatic colorectal cancer. The New England journal of medicine. 2004; 350:2335-2342.

7. Tol J, Dijkstra JR, Klomp M, Teerenstra S, Dommerholt M, Vink-Borger ME, van Cleef PH, van Krieken JH, Punt CJ and Nagtegaal ID. Markers for EGFR pathway activation as predictor of outcome in metastatic colorectal cancer patients treated with or without cetuximab. European journal of cancer. 2010; 46:1997-2009.

8. Koopman M, Venderbosch S, van Tinteren H, Ligtenberg MJ, Nagtegaal I, Van Krieken JH and Punt CJ. Predictive and prognostic markers for the outcome of chemotherapy in advanced colorectal cancer, a retrospective analysis of the phase III randomised CAIRO study. European journal of cancer. 2009; 45:1999-2006.

9. Cancer Genome Atlas N. Comprehensive molecular characterization of human colon and rectal cancer. Nature. 2012; 487:330-337.

10. Seshagiri S, Stawiski EW, Durinck S, Modrusan Z, Storm EE, Conboy CB, Chaudhuri S, Guan Y, Janakiraman V, Jaiswal BS, Guillory J, Ha C, Dijkgraaf GJ, et al. Recurrent R-spondin fusions in colon cancer. Nature. 2012; 488:660664.

11. Saltz LB, Clarke S, Diaz-Rubio E, Scheithauer W, Figer A, Wong R, Koski S, Lichinitser M, Yang TS, Rivera F, Couture F, Sirzen F and Cassidy J. Bevacizumab in combination with oxaliplatin-based chemotherapy as firstline therapy in metastatic colorectal cancer: a randomized phase III study. Journal of clinical oncology. 2008; 26:2013-2019.

12. Van Cutsem E, Kohne CH, Hitre E, Zaluski J, Chang Chien CR, Makhson A, D'Haens G, Pinter T, Lim R, Bodoky G, Roh JK, Folprecht G, Ruff P, et al. Cetuximab and chemotherapy as initial treatment for metastatic colorectal cancer. The New England journal of medicine. 2009; 360:1408-1417.

13. Okamoto K, Kodama K, Takase K, Sugi NH, Yamamoto $\mathrm{Y}$, Iwata $\mathrm{M}$ and Tsuruoka A. Antitumor activities of the targeted multi-tyrosine kinase inhibitor lenvatinib (E7080) against RET gene fusion-driven tumor models. Cancer letters. 2013; 340:97-103.

14. Drilon A, Wang L, Hasanovic A, Suehara Y, Lipson D, Stephens P, Ross J, Miller V, Ginsberg M, Zakowski MF, Kris MG, Ladanyi M and Rizvi N. Response to Cabozantinib in patients with RET fusion-positive lung adenocarcinomas. Cancer discovery. 2013; 3:630-635.

15. Yokota K, Sasaki H, Okuda K, Shimizu S, Shitara M, Hikosaka Y, Moriyama S, Yano M and Fujii Y. KIF5B/ RET fusion gene in surgically-treated adenocarcinoma of the lung. Oncol Rep. 2012; 28:1187-1192.

16. Ju YS, Lee WC, Shin JY, Lee S, Bleazard T, Won JK, Kim YT, Kim JI, Kang JH and Seo JS. A transforming KIF5B and RET gene fusion in lung adenocarcinoma revealed from whole-genome and transcriptome sequencing. Genome research. 2012; 22:436-445.

17. McPherson A, Hormozdiari F, Zayed A, Giuliany R, Ha G, Sun MG, Griffith M, Heravi Moussavi A, Senz J, Melnyk N, Pacheco M, Marra MA, Hirst M, et al. deFuse: an algorithm for gene fusion discovery in tumor RNA-Seq data. PLoS Comput Biol. 2011; 7:e1001138.

18. Ge H, Liu K, Juan T, Fang F, Newman M and Hoeck W. FusionMap: detecting fusion genes from next-generation sequencing data at base-pair resolution. Bioinformatics. 2011; 27:1922-1928.

19. Martin-Zanca D, Hughes SH and Barbacid M. A human oncogene formed by the fusion of truncated tropomyosin and protein tyrosine kinase sequences. Nature. 1986; 319:743-748.

20. Wilton SD, Eyre H, Akkari PA, Watkins HC, MacRae C, Laing NG and Callen DC. Assignment of the human a-tropomyosin gene TPM3 to 1q22- $\rightarrow$ q23 by fluorescence in situ hybridisation. Cytogenet Cell Genet. 1995; 68:122124.

21. Vaishnavi A, Capelletti M, Le AT, Kako S, Butaney M, Ercan D, Mahale S, Davies KD, Aisner DL, Pilling AB, Berge EM, Kim J, Sasaki H, et al. Oncogenic and drugsensitive NTRK1 rearrangements in lung cancer. Nature medicine. 2013; 19:1469-1472.

22. Wiesner T, He J, Yelensky R, Esteve-Puig R, Botton T, Yeh I, Lipson D, Otto G, Brennan K, Murali R, Garrido M, Miller VA, Ross JS, et al. Kinase fusions are frequent in Spitz tumours and spitzoid melanomas. Nat Commun. 
2014; 5:3116.

23. Ross JS, Wang K, Gay L, Al-Rohil R, Rand JV, Jones DM, Lee HJ, Sheehan CE, Otto GA, Palmer G, Yelensky R, Lipson D, Morosini D, et al. New routes to targeted therapy of intrahepatic cholangiocarcinomas revealed by nextgeneration sequencing. Oncologist. 2014; 19:235-242.

24. Kim J, Lee Y, Cho HJ, Lee YE, An J, Cho GH, Ko YH, Joo KM and Nam DH. NTRK1 fusion in glioblastoma multiforme. PLoS One. 2014; 9:e91940.

25. Greco A, Miranda C and Pierotti MA. Rearrangements of NTRK1 gene in papillary thyroid carcinoma. Mol Cell Endocrinol. 2010; 321:44-49.

26. Creancier L, Vandenberghe I, Gomes B, Dejean C, Blanchet JC, Meilleroux J, Guimbaud R, Selves J and Kruczynski A. Chromosomal rearrangements involving the NTRK1 gene in colorectal carcinoma. Cancer letters. 2015; 365:107-111.

27. Kohno T, Ichikawa H, Totoki Y, Yasuda K, Hiramoto M, Nammo T, Sakamoto H, Tsuta K, Furuta K, Shimada Y, Iwakawa R, Ogiwara H, Oike T, et al. KIF5B-RET fusions in lung adenocarcinoma. Nature medicine. 2012; 18:375377.

28. Lee JS, Hirsh V, Park K, Qin S, Blajman CR, Perng RP, Chen YM, Emerson L, Langmuir P and Manegold C. Vandetanib Versus placebo in patients with advanced non-small-cell lung cancer after prior therapy with an epidermal growth factor receptor tyrosine kinase inhibitor: a randomized, double-blind phase III trial (ZEPHYR). Journal of clinical oncology. 2012; 30:1114-1121.

29. Dy GK, Hillman SL, Rowland KM, Jr., Molina JR, Steen PD, Wender DB, Nair S, Mandrekar S, Schild SE, Adjei AA and North Central Cancer Treatment Group Study N. A front-line window of opportunity phase 2 study of sorafenib in patients with advanced nonsmall cell lung cancer: North Central Cancer Treatment Group Study N0326. Cancer. 2010; 116:5686-5693.

30. Spigel DR, Burris HA, 3rd, Greco FA, Shipley DL, Friedman EK, Waterhouse DM, Whorf RC, Mitchell RB, Daniel DB, Zangmeister J, Bass JD and Hainsworth JD. Randomized, double-blind, placebo-controlled, phase II trial of sorafenib and erlotinib or erlotinib alone in previously treated advanced non-small-cell lung cancer. Journal of clinical oncology. 2011; 29:2582-2589.

31. $\mathrm{Wu}$ TD and Nacu S. Fast and SNP-tolerant detection of complex variants and splicing in short reads. Bioinformatics. 2010; 26:873-881.

32. Trapnell C, Pachter L and Salzberg SL. TopHat: discovering splice junctions with RNA-Seq. Bioinformatics. 2009; 25:1105-1111.

33. Ju YS, Kim JI, Kim S, Hong D, Park H, Shin JY, Lee S, Lee WC, Kim S, Yu SB, Park SS, Seo SH, Yun JY, et al. Extensive genomic and transcriptional diversity identified through massively parallel DNA and RNA sequencing of eighteen Korean individuals. Nat Genet. 2011; 43:745-752.

34. Lipson D, Capelletti M, Yelensky R, Otto G, Parker A,
Jarosz M, Curran JA, Balasubramanian S, Bloom T, Brennan KW, Donahue A, Downing SR, Frampton GM, et al. Identification of new ALK and RET gene fusions from colorectal and lung cancer biopsies. Nature medicine. 2012; 18:382-384.

35. Reich M, Liefeld T, Gould J, Lerner J, Tamayo P and Mesirov JP. GenePattern 2.0. Nat Genet. 2006; 38:500-501.

36. Trapnell C, Williams BA, Pertea G, Mortazavi A, Kwan G, van Baren MJ, Salzberg SL, Wold BJ and Pachter L. Transcript assembly and quantification by RNA-Seq reveals unannotated transcripts and isoform switching during cell differentiation. Nat Biotechnol. 2010; 28:511-515.

37. Raychaudhuri S, Stuart JM and Altman RB. Principal components analysis to summarize microarray experiments: application to sporulation time series. Pac Symp Biocomput. 2000:455-466.

38. International HapMap C, Altshuler DM, Gibbs RA, Peltonen L, Altshuler DM, Gibbs RA, Peltonen L, Dermitzakis E, Schaffner SF, Yu F, Peltonen L, Dermitzakis E, Bonnen $\mathrm{PE}$, et al. Integrating common and rare genetic variation in diverse human populations. Nature. 2010; 467:52-58. 